\title{
Using Auditory Displays to Efficiently Present Psychological Research Findings: A Brief Introduction
}

\author{
D. Angus Clark \\ Department of Psychiatry, University of Michigan
}

Karen Brown

Department of Music, University of Pittsburgh

Author Note: Correspondence regarding this manuscript should be addressed to D. Angus

Clark, Department of Psychiatry, University of Michigan, 4250 Plymouth Rd., Ann Arbor, MI 48109. E-mail: cladavid@ med.umich.edu. 


\begin{abstract}
Psychological research is increasingly reliant on high-dimensionality data and complex statistical methods. This offers many benefits, but also entails many challenges. One challenge is that of effective communication. It can be difficult to clearly and concisely communicate trends from series of advanced models and multidimensional data through tables, figures, and narration alone. Auditory displays (AD), or the sound-based representations of data and statistical results, are one tool for helping to address this challenge. AD provide a means of quickly conveying a large quantity of information in a way that is accessible to professional and lay audiences alike. Although AD are often used in other scientific disciplines, they are largely absent from the field of psychology. This article thus provides an introduction to AD and its potential advantages for psychological researchers. As part of this effort, a series of accessible AD are included that readers can interact with and apply to their own work. The overarching goal is to provide researchers with another, somewhat novel, tool for effectively disseminating their research. Keywords: Auditory Displays, Sonification, Psychological Science, Dissemination
\end{abstract}




\section{Introduction}

Complex data and sophisticated statistical methods are increasingly common in the field of psychology. For example, genome-wide association studies (GWAS; Hirschhorn \& Daly, 2005), brain imaging studies (Camara, Marco-Pallares, Munte, \& Rodriguez-Fornells, 2009), and Monte Carlo simulation studies (Bandalos \& Gagne, 2012) all entail the collection and analysis of large quantities of multifaceted data. Furthermore, increasingly advanced statistical methods are continually being developed that provide ever more fine-grained (and at times, difficult to interpret) information about trends in data. Denser data and more detailed statistical models help support stronger inferences about psychological phenomenon, though their use also entails many challenges. One of these challenges is that of effective communication (Tufte, 1997; Tufte, 2001; Wainer, 1997). Even important discoveries and promising new analytic techniques will struggle to gain traction if they cannot be clearly and concisely communicated to the research community, as well as lay audiences and students.

Auditory Displays (AD), or sound-based representations of research findings, and are one tool for effectively disseminating complex, multidimensional results to a broad audience. AD are not commonly used with psychological research, however. The overarching goal of this paper is therefore to show how AD can be developed in the context of psychological research, and why this can be useful. First, a brief introductory overview of AD is provided. Second, a series of increasingly complex, interactive examples of $\mathrm{AD}$ are presented; these illustrative $\mathrm{AD}$ are freely accessible for researchers to apply in their own work. Third, practical considerations for incorporating $\mathrm{AD}$ into research are discussed. 


\section{Auditory Displays and Psychological Research}

In auditory displays (AD), features of data or statistical models are connected to various auditory elements such as pitch, timbre, and tempo. Through this process analytic findings are essentially turned in to a dynamic quasi-instrument that users can manipulate to explore features of the statistical model and its results (Hermann \& Ritter, 2002). Different dimensions of analysis can be actively introduced or subtracted from the auditory environment, and the effects of these manipulations can be heard in real time. AD are therefore able to efficiently communicate intricate findings in a broad, holistic manner that would be difficult to reproduce narratively or visually (Bronkhorst, Veltman, \& van Breda, 1996).

$\mathrm{AD}$ are used across a diverse array of scientific disciplines because of these strengths (e.g., Childs \& Pulkki, 2003; Cittaro et al., 2016; Speeth, 1961). However, AD are less common in the field of psychology (though there have been some applications of $\mathrm{AD}$ in neuroscience; e.g., Bidelman, 2018), despite earlier efforts to promote AD (e.g., Flowers, 2005; Flowers, Buhman, \& Turnage, 2005). Since this prior advocacy the technologies to perform complex analyses, and create AD, have both improved greatly (Neuhoff, 2019). Accordingly, there are presently more potential applications of $\mathrm{AD}$ in psychological research, and researchers have greater flexibility in generating AD.

In the following section we attempt to make the development, functionality, and advantages of $\mathrm{AD}$ more concrete by presenting a series of illustrative $\mathrm{AD}$. Before proceeding though, it is important to highlight a distinction between AD for presenting raw data versus the results from one or a series of statistical models. Historically, most applications of AD have focused on presenting trends in raw data. A more novel application of $\mathrm{AD}$, and the sort illustrated here, is the use of $\mathrm{AD}$ to present one or a series of statistical models by audibly 
representing parameter estimates, model implied trends, or both (Neuhoff, 2019). This modelbased $\mathrm{AD}$ is particularly relevant for psychological research as studies often include several models that are either being compared against one another, or presented in succession to answer interrelated questions (e.g., the same basic model with different outcomes). Indeed, AD provide a means of efficiently capturing multidimensional trends both within and between models with a single representation.

\section{Example Auditory Displays: Linear Regression with Multiple Predictors and Outcomes}

Three AD are presented in turn. The first is an $\mathrm{AD}$ for a simple linear regression model with a single predictor and a single outcome. The second AD is for a single multiple regression model with five predictors. The third AD is for five multiple regression models, each with five predictors. All AD presented here, along with more detailed user instructions and sample programming interfaces, can be accessed online at: https://osf.io/hwfc9/. Users can manipulate these $\mathrm{AD}$, impute the results from their own models, and should feel free to use these AD for both exploring and presenting their own research.

The AD here were all programmed in the MaxMSP software (Cycling ‘74, 2020). MaxMSP is one of the most popular programs for creating $\mathrm{AD}$, and free versions are available for using the $\mathrm{AD}$ presented here at the Cycling' 74 website: https://cycling74.com/. Although a full description of programming in MaxMSP is beyond the scope of the current paper, it is an accessible, object-based programming environment with an intuitive graphical user interface. That is, within the MaxMSP interface users move and interact with a variety of "objects" -which are parcels of alterable code similar to functions in "R" (R Core Team, 2020) -- to build their own sound systems; the look and feel is somewhat reminiscent of constructing path diagrams for displaying and estimating structural equation models. Annotated MaxMSP 
programming interfaces for the three linear regression examples (along with a glossary for the more novel concepts in these files) can be found in the online supplement (https://osf.io/hwfc9/). For a more thorough introduction to MaxMSP, we recommend starting with the extensive documentation on the Cycling "74 website (specifically under the "Learn" tab; (https://cycling74.com/get-started), and Christopher Dobrian's “Max Cookbook" website (Dobrian, 2017).

\section{Example 1: Single Predictor Regression Auditory Display}

The first example AD is for the single predictor linear regression model:

$$
\mathrm{Y}=\beta 0+(\beta 1 * \mathrm{X} 1)+\varepsilon
$$

where $\mathrm{Y}$ is a dependent variable, $\beta 0$ is a constant (i.e., intercept), $\beta 1$ is a regression coefficient indexing the extent to which $\mathrm{Y}$ changes in response to changes in the independent variable (X1), and $\varepsilon$ is a residual term. The first step of developing an AD is determining which dimensions of the analysis need to be represented audibly. In this example the dimensions of interest are the outcome variable ( $\mathrm{Y}$ in Equation 1), and how the outcome changes in responses to changes in the predictor variable $((\beta 1 * \mathrm{X} 1)$ in Equation 1$)$.

The second step of developing an AD is identifying methods for audibly distinguishing both within and between dimensions of the AD. Here, this involves determining what the general sound associated with the outcome variable will be, and how it will change in response to changes in the predictor variable. Dimensions can be distinguished, for example, through pitch, the tempo/speed of tone repetitions, speaker-panning (i.e., alternating between right and left speakers), and timbre (i.e., the quality of the sound). In this example Y is represented by a single, 
repeating tone, and as $\mathrm{Y}$ changes in response to changes in $\mathrm{X} 1$ the tone and speed of these repetitions change. As $\mathrm{Y}$ increases in response to X1 the tone becomes higher-pitched, and the repetitions become more rapid; as $\mathrm{Y}$ decreases in response to $\mathrm{X} 1$ the tone becomes lower pitched, and the repetitions become slower. The change in tone and repetition speed is directly tied to the changes in Y such that -- holding the intercept ( $\beta 0$ in Equation 1$)$ constant -- larger regression coefficients, and/or larger values of X1, lead to more dramatic auditory changes.

A diagram of the interface for this auditory display is presented in Figure 1. The "AD On/Off' button turns the AD on or off, while preserving the settings entered during that session. The "Master Audio" button turns the audio for the entire AD on or off (acting as a mute button). The "AD Volume" slider sets how loud or soft the entire AD is. The "Regression Coefficient" box is where the regression coefficient ( $\beta 1$ in Equation $1 ; \beta 1=.35$ in Figure 1 ) is entered. Notably, to simplify these examples the underlying regression equations for all AD here are based on a standardized solution in which the intercept is 0 , and the regression coefficients reflect standard deviation units; intercept values can be added to AD if necessary. The slider above the "Regression Coefficient" box can be manipulated to increase or decrease the value of the predictor variable (X1 in Equation 1); the value of $\mathrm{X} 1$ being fed into the $\mathrm{AD}$ will be displayed in the "Predictor Value" box $(\mathrm{X} 1=1.5$ in Figure 1). The "Outcome" box displays the outcome value ( $\mathrm{Y}$ in Equation 1) given the regression coefficient and predictor value $(\mathrm{Y}=.53$ in Figure 1). For this example AD, we have included a series of preset regression coefficients corresponding to small $(\beta 1=.15)$, moderate $(\beta 1=.35)$, large $(\beta 1=.50)$, and very large $(\beta 1=1$; the maximum allowable coefficient for this AD) effects in the "Preset Coefficients" box; the "clear all" button sets both the regression coefficient and predictor values to 0 .

\section{Example 2: Multiple Regression Auditory Display}


The second $\mathrm{AD}$ extends the single predictor linear regression $\mathrm{AD}$ to the multiple linear regression model with five predictors (the fifth predictor variable is binary):

$$
\mathrm{Y}=\beta 0+(\beta 1 * \mathrm{X} 1)+(\beta 2 * \mathrm{X} 2)+(\beta 3 * \mathrm{X} 3)+(\beta 4 * \mathrm{X} 4)+(\beta 5 * \mathrm{X} 5)+\varepsilon
$$

where $\mathrm{Y}$ is the dependent variable, $\beta 0$ is a constant (i.e., intercept), $\beta 1-\beta 5$ are regression coefficients indexing how $\mathrm{Y}$ changes in response to different independent variables (X1-X5) holding all others constant, and $\varepsilon$ is a residual term. The general structure of the auditory mapping here is the same as the first example, with the main difference being that now $\mathrm{Y}$ can be affected by multiple independent variables. That is, the dimensions that need to be linked to auditory elements are the outcome variable ( $\mathrm{Y}$ in Equation 2), and how the outcome changes in responses to changes in any individual predictor variable, or combination of predictor variables (X1-X5 in Equation 2). Once again $\mathrm{Y}$ is represented via a single repeating tone; as $\mathrm{Y}$ increases the tone becomes higher-pitched, and the repetitions become more rapid, and vice-versa. Importantly, the effect of any individual predictor variable can be examined both in isolation, and in combination with the other predictor variables.

The interface for the multiple regression $\mathrm{AD}$ is a straightforward extension of the $\mathrm{AD}$ for the single predictor regression model, and is depicted in Figure 2. All of the overlapping features function as described above. The main difference is that now there are five boxes for regression coefficients ( $\beta 1$ - $\beta 5$ in Equation 2), and five sliders for manipulating the values of the predictor variables (X1-X5 in Equation 2). In Figure 2 the regression coefficients are set to $\beta 1=.5, \beta 2=-$ $.25, \beta 3=.10, \beta 4=.05$, and $\beta 5=-.30$; the predictor values are set to $\mathrm{X} 1=.30, \mathrm{X} 2=.10, \mathrm{X} 3=-$ $.15, \mathrm{X} 4=.50$, and $\mathrm{X} 5=1$. There are two new features worth highlighting in this AD. The first is 
the "Magnification" box, which can be used to increase the sensitivity of the AD to changes along a narrower range of the outcome variable. This can be used to make effects within a certain range (e.g., between the mean and one standard deviation) more pronounced. This is similar to changing the scale of the $\mathrm{Y}$ axis in a graph in order to emphasize trends within a certain range. Second, the "Zero Reference Volume" slider introduces a distinct repeating tone with a pitch and tempo corresponding to an outcome value of 0 ; this provides an optional, auditory reference point to compare the effects of different variables against.

\section{Example 3: Multiple Outcomes-Multiple Regression Auditory Display}

The third AD expands the multiple regression $\mathrm{AD}$ to accommodate the five, side-by-side multiple regression models:

$$
\begin{aligned}
& Y_{1}=\beta 0_{1}+\left(\beta 1_{1} * X 1_{1}\right)+\left(\beta 2_{1} * X 2_{1}\right)+\left(\beta 3_{1} * X 3_{1}\right)+\left(\beta 4_{1} * X 4_{1}\right)+\left(\beta 5_{1} * X 5_{1}\right)+\varepsilon \\
& Y_{2}=\beta 0_{2}+\left(\beta 1_{2} * X 1_{2}\right)+\left(\beta 2_{2} * X 2_{2}\right)+\left(\beta 3_{2} * X 3_{2}\right)+\left(\beta 4_{2} * X 4_{2}\right)+\left(\beta 5_{2} * X 5_{2}\right)+\varepsilon \\
& Y_{3}=\beta 0_{3}+\left(\beta 1_{3} * X 1_{3}\right)+\left(\beta 2_{3} * X 2_{3}\right)+\left(\beta 3_{3} * X 3_{3}\right)+\left(\beta 4_{3} * X 4_{3}\right)+\left(\beta 5_{3} * X 5_{3}\right)+\varepsilon \\
& Y_{4}=\beta 0_{4}+\left(\beta 1_{4} * X 1_{4}\right)+\left(\beta 2_{4} * X 2_{4}\right)+\left(\beta 3_{4} * X 3_{4}\right)+\left(\beta 4_{4} * X 4_{4}\right)+\left(\beta 5_{4} * X 5_{4}\right)+\varepsilon \\
& Y_{5}=\beta 0_{5}+\left(\beta 1_{5} * X 1_{5}\right)+\left(\beta 2_{5} * X 2_{5}\right)+\left(\beta 3_{5} * X 3_{5}\right)+\left(\beta 4_{5} * X 4_{5}\right)+\left(\beta 5_{5} * X 5_{5}\right)+\varepsilon
\end{aligned}
$$

where the primary dimensions of interest are now the outcome variables $\left(\mathrm{Y}_{1}\right.$ through $\mathrm{Y}_{5}$ in Equations 3 through 7), and how the outcomes change in responses to changes in any of their specific predictor variables, or combination of predictor variables (X1-X5 in Equations 3 through 7). The key challenges in moving from Example 2 to Example 3 are differentiating between the outcome variables, and enabling the coherent activation of multiple outcomes at once. Here, this 
is accomplished by mapping the outcomes to distinct repeating tones differentiated by timbre and/or octave, and by adding a set of specific volume controls for each outcome.

The AD for each of these five multiple regression models functions the same as the single multiple regression AD described above. This can be seen more clearly in Figure 3, which depicts the interface for the multiple outcomes multiple regression AD. In the top half of this diagram five versions of the same interface depicted in Figure 2 are simply reproduced side by side (albeit on a smaller scale). The main additions to this interface are the extra "Outcome Variable" volume sliders. These can be used to select which model, or models, are being heard, and determine the relative volume of each. Notably, given the amount of parameters in this AD we also provide a companion interface online specifically for quickly entering in values for the various regression equations and saving them for future use. Up to 10 sets of regression coefficients (and predictor values -- though the sliders can still be used to change these values in the main interface) can be saved and quickly recalled so users do not need to re-calibrate the entire AD before every use. This feature is described more fully in the online supplement. Although a straightforward extension of the first two examples, the multiple outcomes multiple regression $\mathrm{AD}$ is a highly flexible $\mathrm{AD}$ that can convey the effects of multiple predictors on up to five (the AD still works if models have less than 5 predictors) different outcomes individually, or together. Indeed, this AD can be used to convey the effects of the same set of predictors on multiple outcomes, the effects of multiple sets of predictors on the same outcome, the effects of two distinct sets of predictors on two distinct outcomes, and more. The specific mapping of the five outcomes to distinct sonic qualities helps ensure that when multiple models are activated it is possible to hear how each variable is changing in response to its predictor variables. That is, using the outcome-specific volume sliders it is possible to precisely control the 
extent to which different outcomes are emphasized against each other for more effective comparisons and data explorations.

\section{Example Auditory Display in Real Data: Predicting Parental Disagreement}

As one final example to further demonstrate the process of developing an $\mathrm{AD}$, and their benefits, we briefly present an $\mathrm{AD}$ that was constructed to more effectively convey the results from a real investigation into predictors of informant disagreement (a more detailed description of this study and the corresponding AD can be found online: https://osf.io/hwfc9/). This AD is specifically based on the 2017 paper by Clark, Durbin, Donnellan and Neppl, "Internalizing Symptoms and Personality Traits Color Parental Reports of Child Temperament". The aim of this study was to investigate why parents' ratings of child personality often disagree by fitting a series of conditional trifactor models (Bauer et al., 2013). These models include latent factors that capture consensus, or agreement, in parents' views of child personality, and individual parents' unique perspective on child personality (with one factor for mothers, and one for fathers). To examine predictors of agreement and disagreement, the consensus and unique perspective factors were regressed on different parent characteristics. The main analyses were thus essentially a series of regression models in which the outcomes were latent, however, across analyses it was necessary to account for two parents, multiple parent characteristics, three different child personality traits, and three latent outcome variables of interest.

In developing an $\mathrm{AD}$ for these analyses, the dimensions of interest were the child traits, agreement versus disagreement, and the effects of mothers' and fathers' characteristics. The different personality traits were represented using different timbres and pitch ranges such that each trait was distinguishable by its own unique sound. Within each trait, mothers' and fathers' unique ratings were represented by successively presenting two separate pitches that were 
differentiated by timbre and/or speaker orientation (i.e., left versus right). The consensus rating was represented by the speed at which these two pitches alternated.

Finally, the effects of the parent characteristics on unique and consensus ratings (disagreement and agreement) were incorporated into the $\mathrm{AD}$ via changes in pitch and tempo. When an attribute increases a unique score, the pitch rises, when it decreases a unique score, the pitch lowers. When an attribute increases a consensus scores, the speed of the alternation between maternal and paternal tones becomes quicker, whereas when it decreases consensus ratings, the vacillations become slower. Altogether it is thus possible to simultaneously hear the effects of a parental attribute on maternal unique ratings, paternal unique ratings, and consensus ratings, with more extreme parent pitches denoting greater disagreement.

The AD for the trifactor model (TMAD), and more detailed background/user instructions, can be accessed at: https://osf.io/hwfc9/. The basic operation for the TMAD is consistent with the simpler examples presented above. Users can input their own hypothetical or real regression coefficients into the TMAD as well, but to explore the Clark et al. (2017) findings the default settings can be used. After turning the audio on it is possible to change the volume of each child personality trait so that users can listen to results for one trait at a time, or consider multiple traits simultaneously.

\section{Practical Considerations in Auditory Display}

The AD presented here demonstrate how $\mathrm{AD}$ can distill one or more statistical models down to a manageable auditory environment to quickly and effectively communicate an otherwise large and complex set of results. Developing effective AD requires some thought, however, and so here we briefly highlight some of the major questions and challenges that those interested in $\mathrm{AD}$ must consider. To reiterate, the two major decision points in constructing an 
$\mathrm{AD}$ are 1) which dimensions of an analysis need to be sonically represented, and 2) what is the most effective way to represent those dimensions.

\section{Which Analytic Dimensions to Represent in an AD?}

Despite the technical capability of AD to represent a virtually unlimited number of dimensions, parsimony is preferred to ensure that the translation of data to sound is as direct and clear as possible. That is, not every aspect of every model needs to be included in an AD, with the number of reasonable dimensions for a given application dependent on the nature of the analyses, and the presentation goals. Researchers should generally focus on the dimensions of their results that are difficult to clearly and concisely describe in text and with figures, and/or that can help holistically clarify trends across analyses. Some typical features of an analysis that might be considered for an $\mathrm{AD}$ include: the effects of different types of predictor variables (e.g., multiple informant reports) with and without covariates, how different types of outcome variables change in response to different types of predictors, or the effect of a large set of predictor variables on an outcome variable across distinct groups.

Furthermore, although the $\mathrm{AD}$ presented here were temporally static, interactive sonic environments, the auditory modality is well suited to presenting trends across time. For example, it would be possible to represent the results from a latent growth model in an AD that conveyed both how different individuals, or groups of individuals, generally change over time, and how these trends are affected by both time invariant and time varying predictor variables. The MaxMSP software handily allows visual streams alongside auditory streams too, meaning that dynamic visual displays of trends over time can be included alongside the auditory representations. This is noteworthy as the combination of visual and auditory presentation can be more effective than any single presentation modality alone (e.g., Koteskey et al., 2012). 


\section{How to Represent Analytic Dimensions in an AD?}

The number and nature of model features selected for inclusion in an AD is directly related to the second major concern, that is, how are dimensions are going to be audibly represented (e.g., via pitch, tempo, panning, timbre)? For example, the changing timbre of a sound could mean one thing, and changing pitch another. In the regression examples changes in the outcomes were represented by both changes in pitch and speed. This coupling of multiple sonic traits to a single variable can help emphasize specific trends with a greater degree of clarity. However, in more complex AD, such as in the TMAD, the sharp distinction between the meaning of speed and pitch is vital for the preservation of clarity between the consensus and individual informant factors. There is thus often a tradeoff between making any one dimension of the AD highly salient (e.g., via multiple sonic characteristics), and representing multiple dimensions simultaneously.

To the greatest extent possible representations in an AD should be thematically consistent with the dimensions they are meant to represent, which can help users more quickly understand the AD. For example, higher outcome values here were associated with higher pitches, and more rapid tempos. Similarly, in the TMAD, the tone associated with the positive emotionality trait is brighter than the tone associated with the negative emotionality trait. The best mapping may not always be intuitive like this, however. Even when there is not a clearly intuitive mapping the goal of providing an auditory environment that can be quickly understood remains vital. Clearly defined analytic dimensions, and salient, distinct representations of those dimensions, are the key ingredients of effective AD. Like poorly designed graphs or tables, AD with unclearly defined dimensions undermine comprehension. This in turn undermines the primary advantage of $\mathrm{AD}$, which is clearly and concisely presenting multifaceted results. Indeed, 
thanks to the distinct representations in the multiple outcomes-multiple regression AD it was possible to reduce the effects of 25 different predictor variables to 5 broad, easily identifiable sonic dimensions.

\section{Additional Considerations}

More complex AD, like the TMAD, that involve the sonification of large quantities of results and include several dimensions will often require some initial instruction to orient users. This can either be written instruction included in an online supplement (as done here), or provided in person during a presentation. Researchers may wish to avoid particularly complicated $\mathrm{AD}$ in certain live settings (e.g., conference symposium) because of this, and rely on simpler examples like those presented here (e.g., moving a small number of sliders to hear changes in one or two outcomes). Notably however, even for more complex AD orientation to the AD will likely not take long, and once achieved pays dividends in terms of how efficiently results can be navigated (Bronkhorst et al., 1996). That is, AD are typically simple to operate, and if effectively constructed will make it very salient as to how users' manipulations effect the sonic environment.

There are other technical considerations when working with AD that extend beyond the conceptualization of the AD itself. AD obviously cannot be included directly in a manuscript, and so will need to be placed online. Some applications, like the example here, may require that users download special software, such as MaxMSP. As noted, the version of MaxMSP needed to operate an existing $\mathrm{AD}$ is free, but this still adds a hurdle to potential users. It may be possible with some applications though to host AD entirely online, either directly or as a video demonstration. For example, Cittaro and colleagues (2016) uploaded many of their genomic based AD to "SoundCloud", a free online service where users can upload and share audio files. 
Hosting an $\mathrm{AD}$ online directly like this however is more restrictive when developers wish to provide users with the ability to input and save their own information. Even when presenting AD in person though there are some practical considerations. For example, using an AD in a conference symposium requires a computer equipped to run it, and quality speakers. The computational burden of AD is typically low though, and speakers are generally present in conference settings regardless.

As a final note, producing particularly effective AD will often be a multi-disciplinary endeavor. Although the programming of $\mathrm{AD}$ is increasingly accessible to any interested party thanks to programs like MaxMSP, those in the musical fields also have the disciplinary expertise necessary to engineer $\mathrm{AD}$ that make the correspondence between results and sound clear. The International Community for Auditory Display (ICAD; https://icad.org/) is home to many individuals with these skills who are often eager for opportunities to collaborate with scientific researchers, and is a useful starting point for building cross-disciplinary collaborations.

\section{Conclusion}

Auditory displays (AD) take the results from one or a series of statistical models and convert them into an interactive auditory environment. Although ADs are used effectively in many other fields, they are less common in the field of psychology. This is unfortunate as AD provide a flexible way to present complex results quickly and comprehensively. Auditory displays facilitate the dissemination of research findings both within the field and beyond it, and to audiences that may lack the methodological background knowledge necessary to understand the particulars of the analyses themselves. Given the importance of dissemination in the research process, auditory displays represent a very useful potential addition to the psychological researcher's toolbox. This overview provided a brief introduction to $\mathrm{AD}$, presenting several 
examples based on popular analytic techniques, examples that are freely available for researchers to use with their own work (https://osf.io/hwfc9/).

Author Contributions: D.A. Clark and K. Brown jointly developed the ideas presented in this manuscript. D. A. Clark conducted the analyses that formed the basis of the TMAD (presented fully in Clark et al., 2017) and wrote the first draft of the manuscript. K. Brown developed the AD softwares and wrote the supporting documentation. Both authors edited the manuscript and approved the final submitted version.

Conflicts of Interest: The author(s) declare that there were no conflicts of interest with respect to the authorship or the publication of this article. 


\section{References}

Bandalos, D. L., \& Gagné, P. (2012). Simulation methods in structural equation modeling. In Handbook of Structural Equation Modeling (pp. 92-110). New York, NY: The Guilford Press.

Bidelman, G. M. (2018). Sonification of scalp-recorded frequency-following responses (FFRs) offers improved response detection over conventional statistical metrics. Journal of Neuroscience Methods, 293, 59-66.

Bauer, D. J., Howard, A. L., Baldasaro, R. E., Curran, P. J., Hussong, A. M., Chassin, L., \& Zucker, R. A. (2013). A trifactor model for integrating ratings across multiple informants. Psychological methods, 18(4), 475.

Bronkhorst, A. W., Veltman, J. A., \& Van Breda, L. (1996). Application of a three-dimensional auditory display in a flight task. Human factors, 38(1), 23-33.

Camara, E., Marco-Pallarés, J., Münte, T. \& Rodríguez-Fornells, A. (2009). Neuroimaging analysis ii: magnetic resonance imaging. In R. E. Millsap \& A. Maydeu-Olivares The SAGE handbook of quantitative methods in psychology (pp. 697-713). London: SAGE Publications Ltd.

Childs, E., \& Pulkki, V. (2003). Using multi-channel spatialization in sonification: a case study with meteorological data. Proceedings of the 2003 International Conference on Auditory Display.

Cittaro, D., Lazarevic, D., \& Provero, P. (2016). Chromas from chromatin: sonification of the epigenome. F1000Research, 5.

Clark, D. A., Durbin, C. E., Donnellan, M. B., \& Neppl, T. K. (2017). Internalizing symptoms 
and personality traits color parental reports of child temperament. Journal of personality, 85(6), 852-866.

Cycling '74. (2020). Max/MSP/Jitter (8.1.2). San Francisco, California: Cycling ’74.

Dobrian, C. (2017). Max Cookbook. https://music.arts.uci.edu/dobrian/maxcookbook/

Flowers, J. H., Buhman, D. C., \& Turnage, K. D. (2005). Data sonification from the desktop: Should sound be part of standard data analysis software? ACM Transactions on Applied Perception (TAP), 2(4), 467-472.

Flowers, J. H. (2005). Thirteen years of reflection on auditory graphing: Promises, pitfalls, and potential new directions. Proceedings of the 2005 International Conference on Auditory Display.

Hermann, T., \& Ritter, H. (2005). Model-based sonification revisited---authors' comments on Hermann and Ritter, ICAD 2002. ACM Transactions on Applied Perception (TAP), 2(4), $559-563$.

Hirschhorn, J. N., \& Daly, M. J. (2005). Genome-wide association studies for common diseases and complex traits. Nature Reviews Genetics, 6(2), 95.

Koteskey, R. W., Wu, S. C., Battiste, V., Wenzel, E. M., Lachter, J., Begault, D. R., ... \& Johnson, W. W. (2012, July). Enhanced Audio for NextGen Flight Decks. In Proc. 4th Int. Conference on Applied Human Factors and Ergonomics.

Neuhoff, J. G. (2019). Is sonification doomed to fail? Georgia Institute of Technology.

R Core Team. (2020). R: A language and environment for statistical computing (3.6.0). Vienna, Austria: R Foundation for Statistical Computing.

Speeth, S. D. (1961). Seismometer sounds. The Journal of the Acoustical Society of America, 33(7), 909-916. 
Tufte, E. R. (1997). Visual explanations: Images and quantities, evidence, and narrative. Graphics Press: Cheshire, CT.

Tufte, E. R. (2001). The visual display of quantitative information ( $2^{\text {nd }}$ edition). Graphics Press: Cheshire, CT.

Wainer, H. (1997). Visual Revelations: Graphical tales of fate and deception from Napoleon Bonaparte to Ross Perot. Psychology Press: East Sussex, UK. 


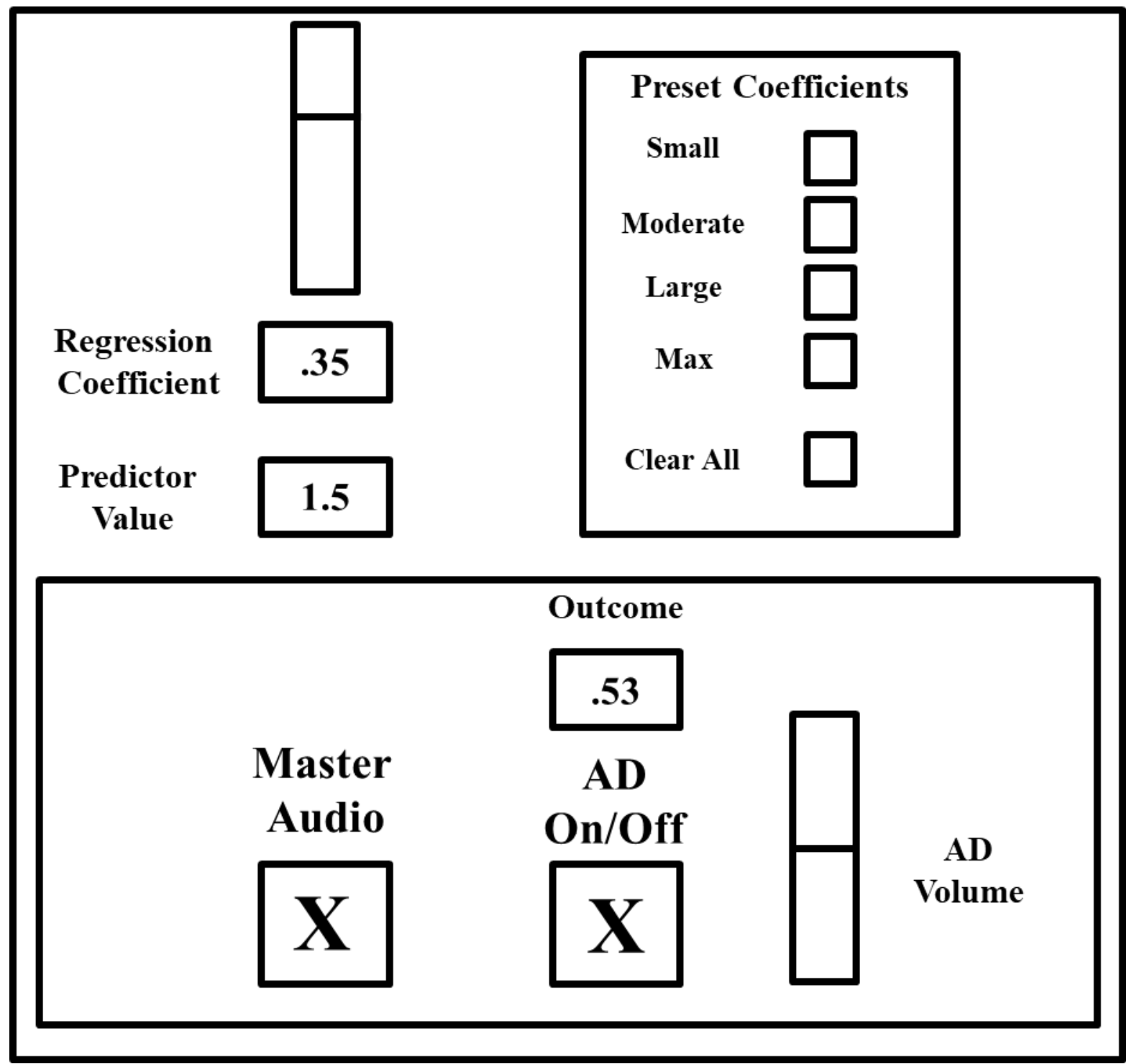

Figure 1. Diagram of the Single Predictor Regression Auditory Display (AD) Control Panel. The auditory display, along with more detailed instructions and a sample programming interface can be found in the online supplemental material. 


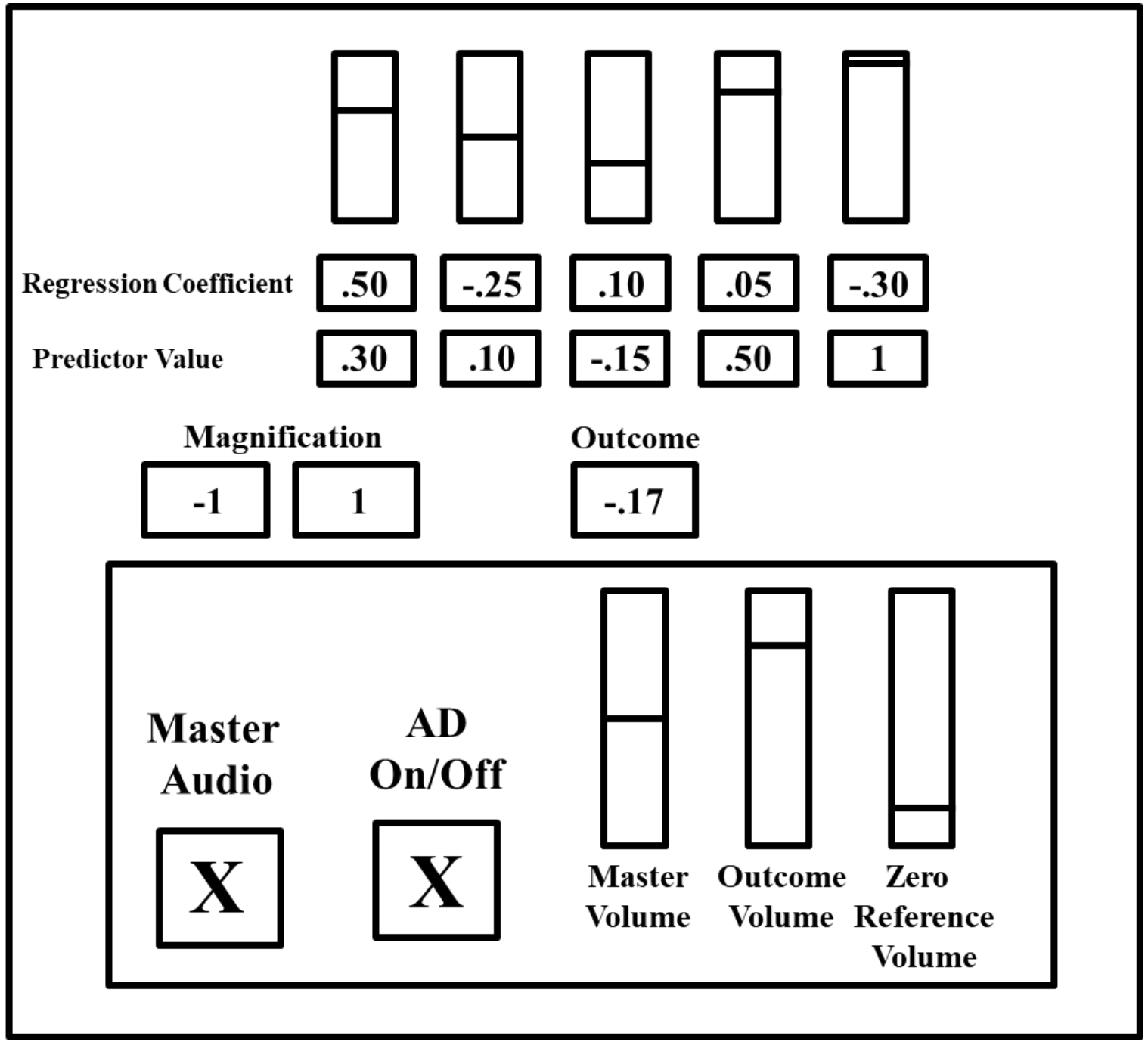

Figure 2. Diagram of the Multiple Regression Auditory Display (AD) Control Panel. The auditory display, along with more detailed instructions and a sample programming interface can be found in the online supplemental material. The preset coefficient options are omitted from the figure for parsimony, but are located above the outcome box and function the same as the preset coefficient options in the single predictor regression AD. 


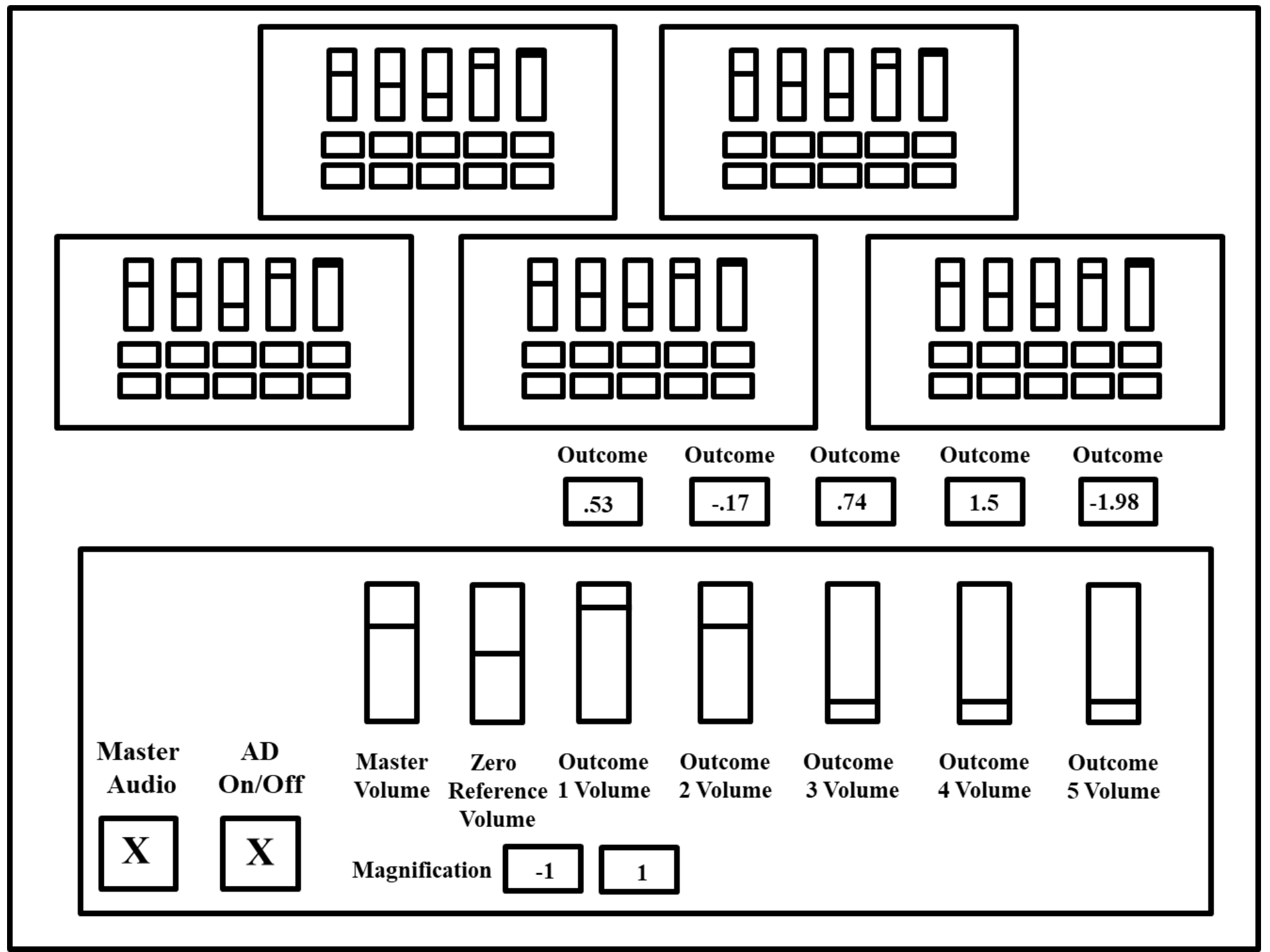

Figure 3. Diagram of the Multiple Outcomes Multiple Regression Auditory Display (AD) Control Panel. The auditory display, along with more detailed instructions and a sample programming interface can be found in the online supplemental material. 